\title{
Electromagnetic moments of relativistic higher spin baryons
}

\author{
V. Pascalutsa* \\ Department of Physics, Flinders University, Bedford Park, SA 5042, Australia
}

We point out a source of ambiguities in the measurements of the electromagnetic moments of spin-3/2 baryons which rely on relativistic Lagrangian models. An anambiguous relation between the parameters of the spin-3/2 electromagnetic Lagrangian and the electromagnetic moments of the spin-3/2 particle exits in general only for on-shell situation, while the measurements are done on "virtual" baryons.

Accurate measurement of electromagnetic (EM) moments of $N^{*}$ resonances could provide an important testing ground for many quark-models and lattice QCD predictions. Because of the short lifetime of the $N^{*}$ resonances such measurements present experimental as well as theoretical challenge. At present one can hope only for indirect measurements. For example, the dipole magnetic moment of the $\Delta^{++, 0}$ isobars is extracted from the observables of radiative pion-nucleon scattering, $\pi^{ \pm}+p \rightarrow \pi^{ \pm}+p+\gamma$ (see, e.g., [1]), while the magnetic moment of the $\Delta^{+}$can possibly soon be measured at MAMI in the radiative pion photoproduction [2], $\gamma+p \rightarrow \pi^{0}+p+\gamma$.

In these reactions the $\gamma \Delta \Delta$ current containing the EM moments is involved only through more complicated mechanisms, see Fig. 1. Moreover, other mechanisms (such as in Fig. 2) may interfere and must be estimated reliably. The necessary theoretical input is usually provided by some effective Lagrangian model based on hadronic degrees of freedom [3 5], where all the relevant mechanisms can be computed, while the parameters other than the EM moments can be fixed from different sources (e.g., by using the same effective Lagrangian for the description of other processes).

Here we shall assume a general form of the effective hadronic Lagrangian restricted only by a few fundamental principles such as Lorentz and gauge invariance, and possibly the conditions due to the approximate chiral symmetry.

We will consider the charge and dipole magnetic moment interactions of the spin-3/2 baryon and show how the parameters of the Lagrangian can unambiguously be related to the magnetic moment of the "real" spin-3/2 particle. For virtual particle this relation is in general ambiguous. This problem is also related to the so-called "off-shell ambiguities" of the covariant spin-3/2 description which generally does not exclude off-shell the unphysical spin-1/2 contributions. (Recently, Machavariani et al. [4] have claimed to get rid of the "off-shell ambiguities" formulation by writing the spin-3/2 propagator in terms of on-shell Rarita-Schwinger vector-spinors. However, in doing so one looses relativistic covariance, see Ref. [6] for details.) Furthermore, by considering the matrix elements of processes

\footnotetext{
${ }^{*}$ Supported by the Australian Research Council (ARC).
} 


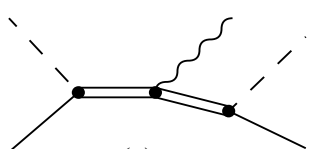

(a)

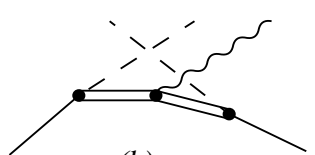

(b)

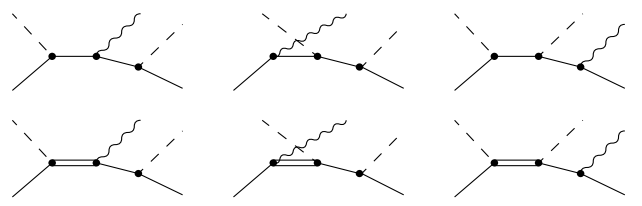

Figure 1. Possible mechanisms of $\pi N$ bremsstrahlung involving the EM moments of the $\Delta$.

Figure 2. Some other relevant mechanisms of $\pi N$ bremsstrahlung.

such as in Fig. 目, we can argue that certain consistency conditions on the form of the $\pi N \Delta$ and $\gamma N \Delta$ couplings may allow us to deal with the "off-shell ambiguities".

To describe the spin-3/2 fields of (decuplet) baryons it is natural to use the covariant Rarita-Schwinger (RS) formalism[7] where the field is a Lorentz vector-spinor $\psi^{\mu}(x)$ with the following free Lagrangian? :

$\mathcal{L}_{\text {free }}=\bar{\psi}_{\mu} \gamma^{\mu \nu \alpha} \partial_{\alpha} \psi_{\nu}-m \bar{\psi}_{\mu} \gamma^{\mu \nu} \psi_{\nu}$

where $m$ is the mass; $\gamma^{\mu \nu}$ and $\gamma^{\mu \nu \alpha}$ is the totally antisymmetrized product of, respectively, two and three gamma-matrices. This Lagrangian leads to the well-known RS propagator:

$S^{\mu \nu}(p)=\frac{\not p+m}{p^{2}-m^{2}+i \varepsilon}\left[-g^{\mu \nu}+\frac{1}{3} \gamma^{\mu} \gamma^{\nu}+\frac{1}{3 m}\left(\gamma^{\mu} p^{\nu}-\gamma^{\nu} p^{\mu}\right)+\frac{2}{3 m^{2}} p^{\mu} p^{\nu}\right]$,

and the following free-field equations:

$(i \not \partial-m) \psi_{\mu}=0, \gamma^{\mu} \psi_{\mu}=0, \partial^{\mu} \psi_{\mu}=0$.

Consider now the EM properties of this field. The electric charge is included via minimal substitution, $\partial_{\alpha} \rightarrow \partial_{\alpha}+i e A_{\alpha}$, into Eq. (11). To explore the dipole magnetic moment we should include all the nonminimal, linear in $F_{\mu \nu}=\partial_{[\mu} A_{\nu]}$ terms. We then have:

$$
\begin{aligned}
\mathcal{L}_{\text {int }}= & e\left\{i \bar{\psi}_{\mu} \gamma^{\mu \nu \alpha} \psi_{\nu} A_{\alpha}+l_{1} \bar{\psi}_{\mu} F^{\mu \nu} \psi_{\nu}+l_{2} \bar{\psi}_{\mu} \gamma \cdot F \cdot \gamma \psi^{\mu}+l_{3} F^{\mu \nu}\left[\bar{\psi}_{\mu} \gamma_{\nu} \gamma \cdot \psi+\bar{\psi} \cdot \gamma \gamma_{\mu} \psi_{\nu}\right]\right. \\
& \left.+l_{4} \bar{\psi} \cdot \gamma(\gamma \cdot F \cdot \gamma) \gamma \cdot \psi+i l_{5} F^{\mu \nu}\left[\bar{\psi}_{\mu} \gamma_{\nu} \gamma \cdot \psi-\bar{\psi} \cdot \gamma \gamma_{\mu} \psi_{\nu}\right]\right\} \equiv \bar{\psi}_{\mu} \Lambda^{\mu \nu} \psi_{\nu} .
\end{aligned}
$$

It is further argued in [8] that $\Lambda^{\mu \nu}$ must be antisymmetric in order to maintain the correct number of spin degrees-of-freedom?: $2 s+1=4$. Hence, one finds $l_{2}=-l_{4}, l_{3}=-2 l_{4}$, and the couplings reduce to

$\Lambda^{\mu \nu}=i e \gamma^{\mu \nu \alpha} A_{\alpha}+e m^{-1}\left[L_{1} F^{\mu \nu}+i L_{2} \gamma^{5} \tilde{F}^{\mu \nu}+i L_{3}\left(F^{\mu \alpha} \gamma_{\alpha} \gamma^{\nu}-\gamma^{\mu} \gamma_{\alpha} F^{\alpha \nu}\right)\right]$,

where $\tilde{F}^{\mu \nu}=\varepsilon^{\mu \nu \varrho \sigma} \partial_{\varrho} A_{\sigma} ; L_{i}$ are dimensionless parameters: $L_{1}=m\left(l_{1}+2 l_{2}\right), L_{2}=-2 m l_{2}$, $L_{3}=m l_{5}$. To relate these parameters to the physical magnetic moment we match this field theory to the soft-photon theorems.

${ }^{2}$ Other forms of free spin-3/2 Lagrangian are frequently used in the literature, but they relate to Eq. (1) by a field redefinition $\psi_{\mu} \rightarrow\left(g_{\mu \nu}+b \gamma_{\mu} \gamma_{\nu}\right) \psi^{\nu}$, with $b \neq-\frac{1}{4}$. Present discussion is fixed to representation (1), however is unchanged for other choices albeit the field redefinition is done in the whole Lagrangian. ${ }^{3}$ This condition does not remove the consistency problem of the minimal coupling [9, but only insures the proper degrees-of-freedom count. 
Consider first the on-shell electromagnetic vertex [obtained from (44) with (5)]:

$$
\begin{aligned}
\varepsilon_{\lambda} \Gamma^{\lambda}(q)= & e m^{-1} \varepsilon_{\lambda} \bar{u}_{\mu}\left(p^{\prime}\right)\left\{-m \gamma^{\mu \nu \lambda}+L_{1}\left(q^{\mu} g^{\lambda \nu}-q^{\nu} g^{\lambda \mu}\right)+i L_{2} \gamma^{5} \varepsilon^{\mu \nu \sigma \lambda} q_{\sigma}\right. \\
& \left.+i L_{3}\left[\left(q^{\mu} \gamma^{\lambda}-q g^{\lambda \mu}\right) \gamma^{\nu}+\gamma^{\mu}\left(q^{\nu} \gamma^{\lambda}-q g^{\lambda \nu}\right)\right]\right\} u_{\nu}(p),
\end{aligned}
$$

where $\varepsilon$ and $q=p^{\prime}-p$ is the polarization and the 4-momentum of the photon; $u$ are the on-shell RS vector-spinors, solutions of Eq. (3) in momentum space.

Using the properties of the on-shell vector-spinor one can see that (i) $L_{3}$ term drops out, (ii) $L_{2}$ term contributes only to higher order in $q$ (basically because $\gamma^{5}$ involves the "small components" of the spinors). Also one can (iii) use various Gordon identities and (iv) verify the following nontrivial identity:

$2 \bar{u}_{\varrho}^{\prime} \gamma_{\mu \nu} u_{\sigma}=\bar{u}_{\varrho}^{\prime}\left(\gamma_{\mu \sigma} u_{\nu}+\gamma_{\sigma \nu} u_{\mu}\right)+\left(\bar{u}_{\mu}^{\prime} \gamma_{\varrho \nu}+\bar{u}_{\nu}^{\prime} \gamma_{\mu \varrho}\right) u_{\sigma}+\mathcal{O}(q)$,

where $u^{\prime} \equiv u\left(p^{\prime}\right), u \equiv u(p), q=p^{\prime}-p$. Using all that, the vertex to the 1st order in $q$ reads

$\varepsilon_{\lambda} \Gamma^{\lambda}=\frac{e}{2 m} \varepsilon_{\lambda} \bar{u}_{\mu}^{\prime}\left[\left(p^{\prime}+p\right)^{\lambda} g^{\mu \nu}-g M_{3 / 2}^{\lambda \sigma, \mu \nu} q_{\sigma}\right] u_{\nu}$,

with $M_{3 / 2}$ being the spin-3/2 Lorentz generator and

$g=\frac{2}{3}\left(1-2 L_{1}\right)$.

On the other hand, according to the soft-photon theorem $g=(\mu / s)(e / 2 m)^{-1}$ is the gyromagnetic ratio; $\mu$ is the magnetic moment.

The same relation is obtained by analyzing the Compton scattering on spin-3/2 particle. For the forward Compton amplitude we obtain[8] (to the 1st order in photon frequency $\omega)$ :

$T_{f i}=\frac{e^{2}}{m} \varepsilon^{\prime} \cdot \varepsilon \bar{u}^{\prime} \cdot u+\frac{i e^{2} \omega}{4 m^{2}}(g-2)^{2} \bar{u}_{\mu}^{\prime} M_{3 / 2}^{\lambda \sigma, \mu \nu} u_{\nu} \varepsilon_{\lambda}^{\prime} \varepsilon_{\sigma}$,

again with the gyromagnetic ratio given by Eq. (9). The use of on-shell conditions such as Eq. (7) is made in obtaining this result.

Obviously, a potential problem arises when one tries to identify the magnetic moment of the virtual particle. One cannot use any of the statements (i)-(iv) to conclude an unambiguous relation between the parameters of the Lagrangian and the physical magnetic moment. In particular, because of the coupling to the spin- $1 / 2$ sector of the RS propagator, $L_{2}$ and $L_{3}$ terms may give contributions of the same order in photon energy as the minimal and $L_{1}$ terms. This problem does not arise if the spin-3/2 propagator could be replaced by a positive-energy spin-3/2 projection operator. However, such replacement can be done only at the expense of loosing the Lorentz and gauge invariance. (Gauge invariance is lost because the EM vertex satisfies the Ward-Takahashi identity for the full RS propagator and not for any truncated form).

${ }^{4}$ Let as remark on the definition of the "anomalous magnetic moment", $\mu_{A}$. It is usually defined as the deviation from the value implied by the minimal coupling: $\mu_{A}=\mu-e / 2 m$. However, taking the point of view that $g=2$ is the universal value for all elementary (pointlike) particles with spin [10], it is more correct to define it as the deviation from the fundamental value, i.e., as follows: $\mu_{A}=\mu-s e m^{-1}$. 
A way to deal with the problem is to consider the complete matrix elements (for graphs like in Fig. 1) and use the specific "consistent" $\pi N \Delta$ and $\gamma N \Delta$ interactions 11, 12. The latter interactions have the property of decoupling the spin- $1 / 2$ sector, and therefore the major source of the off-shell ambiguity should be eliminated. A study in this direction

and development of models for the radiative $\pi N$ scattering and pion photoproduction is in progress [13].

\section{REFERENCES}

1. A. Bosshard et al., Phys. Rev. D44, 1962 (1991).

2. V. Metag et al., in progress.

3. L.A. Kondratyuk and L.A. Ponomarev, Sov. J. Nucl. Phys. 7, 82 (1968); W.E. Fischer and P. Minkowski, Nucl. Phys. B36, 519 (1972); P. Pascual and R. Tarrach, Nucl. Phys. B134, 133 (1978); R. Wittman, Phys. Rev. C 37, 2075 (1988); D. Lin, M.K. Liou, and Z.M. Ding, Phys. Rev. C 44, 1819 (1991).

4. A.I. Machavariani, A. Faessler, and A.J. Buchmann, Nucl. Phys. A646, 231 (1999).

5. D. Drechsel, M. Vanderhaeghen, M. M. Giannini and E. Santopinto, nucl-th/0003035.

6. E. van Faassen and J.A. Tjon, Phys. Rev. C 30, 285 (1984); ibid. 33, 2105 (1986);

E. van Faassen, Ph.D. thesis, University of Utrecht, 1985.

7. W. Rarita and J. Schwinger, Phys. Rev. 60, 61 (1941).

8. S. Deser, V. Pascalutsa, and A. Waldron, hep-th/0003011.

9. K. Johnson and E. C. Sudarshan, Annals Phys. 13, 126 (1961);

G. Velo and D. Zwanziger, Phys. Rev. 186, 1337 (1969).

10. S. Weinberg in "Lectures on Elementary Particles and Quantum Field Theory", Volume 1, Brandeis University Summer Institute 1970 (Deser, Grisaru and Pendleton, editors, M.I.T. Press, Cambridge, 1970).

11. V. Pascalutsa, Phys. Rev. D 58, 096002 (1998).

12. V. Pascalutsa and R. Timmermans, Phys. Rev. C 60, 042201 (1999).

13. R. Timmermans and V. Pascalutsa, in progress. 